\title{
REVISÃO SISTEMÁTICA DA LITERATURA SOBRE DETECÇÃO DE ÁRVORES UTILIZANDO DADOS DE SENSORIAMENTO REMOTO
}

\author{
SYSTEMATIC REVIEW OF LITERATURE ON TREE DETECTION USING REMOTE SENSING
}

\author{
Dyeison Cesar Mlenek ${ }^{1}$, Ana Paula Dalla Corte ${ }^{2}$, Daniel Rodrigues dos Santos ${ }^{3}$, \\ Carlos Roberto Sanqueta ${ }^{4}$ \\ 1,2,3,4 Universidade Federal do Paraná, Curitiba, Paraná, Brasil-dyeison.mlenek@hotmail.com, \\ anapaulacorte@gmail.com,danielsantos@ufpr.br\&sanquetta@ufpr.br
}

\section{RESUMO}

O conhecimento do cenário atual referente formas de detecção de árvores por meio de sensoriamento remoto em plantios florestais é essencial para orientar novos estudos relacionados ao tema. Com as publicações científicas dos principais autores e periódicos do assunto dos últimos 20 anos, espera-se neste estudo delinear os principais tipos de dados, espécies e algoritmos estudados recentemente. Com o objetivo de apresentar o desenvolvimento de pesquisas em situações especificas com determinadas espécies, idades, espaçamento, tipos de dados e algoritmos, visando a contagem de árvores. Após reunir a literatura disponível nas bases selecionadas, Web of Science e a Scopus, foi realizada uma análise bibliométrica. Para a mesma utilizou-se o pacote bibliometrix desenvolvido para a linguagem $\mathrm{R}$, para a filtragem de documentos e representação gráfica dos resultados. Foram encontrados resultados classificados em aceitáveis ou não aceitáveis para estimativas florestais, apresentando uma variada gama de alternativas para obtenção do número de árvores de um povoamento florestal, devido as diferenças entre os tipos de dados e algoritmos utilizados. Conclui-se que atualmente não existe uma metodologia padrão para a detecção de árvores e dificilmente será aplicável um método genérico para a contagem, visto a heterogeneidade das florestas e diferentes tratamentos de dados. Foi ainda observadas limitações quanto ao desenvolvimento de metodologias satisfatórias para plantios de Pinus taeda, regularmente espaçados e com idades próximas ao corte comercial.

PALAVRAS-CHAVE: Bibliometria, Contagem de árvores, Identificação de árvores.

\begin{abstract}
The knowledge of the current scenario regarding the ways of detecting trees in forest plantations is essential to guide new studies related to the theme. With the scientific publications of the main authors and periodicals of the subject of the last 20 years, it is sought to outline the main types of data, species and algorithms studied recently. With the objective of present the development of research in specific situations with certain species, ages, spacing, data types and algorithms, aiming at tree counting. After gathering the available literature, a bibliometric analysis was performed, using the bibliometrix package developed for the $\mathrm{R}$ language, for the filtering of documents and graphic representation of the results. Results were classified as acceptable or not acceptable for forest estimates, presenting a wide range of alternatives to obtain the number of trees in a forest stand due to differences between the data types and algorithms used. It is concluded that there is currently no standard methodology for the detection of trees and it is unlikely that a generic method for counting will be applicable, given the heterogeneity of the forests and different data treatments. Limitations were also observed regarding the development of satisfactory methodologies for regularly spaced Pinus taeda plantations with ages close to commercial cutting
\end{abstract}

KEYWORDS: Bibliometrics, Tree Count, Tree Identification. 


\section{INTRODUÇÃO}

A revisão bibliográfica conforme Webster \& Watson (2011) é considerada um passo inicial para qualquer pesquisa científica, assim como, definir a linha limítrofe da pesquisa que se deseja desenvolver. Para Biolchini et al. (2007), é tratada como um instrumento para mapear trabalhos publicados, para que o pesquisador elabore uma síntese do conhecimento existente sobre o assunto.

De acordo com Ferreira (2010) bibliometria, entendese como uma técnica quantitativa e estatística de medição dos índices de produção científica, baseado em um conjunto de leis e princípios empíricos para o estabelecimento dos fundamentos teóricos.

Para mensurar o nível de desenvolvimento e perspectivas futuras da detecção de árvores em povoamentos florestais por meio de sensoriamento remoto, a consulta a literatura é essencial. Conforme Kocsan et al. (2018), contagens e tamanhos de árvores são informações importantes a serem aplicadas à estimativa de produção e ao planejamento florestal. No estudo de Ok \& Ozdarici (2018), é apontado como um desafio do sensoriamento remoto e da visão computacional a identificação de árvores utilizando métodos automatizados por algoritmos. Portanto, obter identificação automática de árvores, suas localizações, diâmetros e contagens a partir de dados remotamente detectados é uma tarefa útil e desafiadora.

O emprego de Light Detection and Ranging (LiDAR) vem se fazendo presente em estimativas florestais, com destaque na possibilidade recente do uso destes sensores acoplados em Aeronave Remotamente Pilotada (ARP). Silva et al. (2016a) indicaram que o LiDAR tem demostrado a existência de um bom potencial para o inventário florestal ao nível de árvores individuais.

Porém, conforme apontado por Packalén et al. (2011), para a viabilização devem ser analisadas as características, como por exemplo: a idade, o espaçamento e a espécie de cada reflorestamento para alcançar um nível aceitável de acerto do número de árvores, que deve ser maior que $80 \%$ (MOHAN et al., 2017).

Kaartinen et al. (2012) ressaltaram que a detecção de árvores, além de fornecer uma distribuição espacial dos indivíduos do povoamento, reduz a quantidade de trabalho em campo relacionado as estimativas florestais. Porém, conforme Silva et al. (2016a), existe a necessidade de estudos para avaliação e comparação dos custos de aquisição e processamento de dados LiDAR contra o inventário florestal convencional.

O presente trabalho espera obter de um panorama geral da detecção de árvores em povoamentos florestais, com base em dados provenientes de sensoriamento remoto e metodologia de identificação automática de indivíduos, com o objetivo de apresentar o desenvolvimento de pesquisas em situações especificas com determinadas espécies, idades, espaçamento, tipos de dados e algoritmos, visando a identificação de árvores.

\section{MATERIAL E MÉTODOS}

O desenvolvimento desta análise bibliométrica fez uso do pacote bibliometrix, escrito na linguagem $\mathrm{R}$, qual tratase de um conjunto de ferramentas estatísticas para a quantificação e representação gráfica da bibliometria. Esta revisão baseou-se no levantamento e análise das publicações científicas ao longo do tempo relacionadas a identificação de árvores em talhões florestais utilizando a tecnologia LiDAR, aerofotogrametria ou técnicas de sensoriamento remoto.

$\mathrm{Na}$ análise bilbiométrica, foram examinados os tipos de documentos publicados (artigos, artigos em revistas, artigos em conferências, erratas e revisões), as palavras chaves propostas pelo autor, as Keywords Plus que tratam de terminologias derivadas dos títulos originais das obras, a multi-autoria que trata de documentos escritos por mais de um pesquisador, e o índice de colaboração que consiste na média de coautores nos documentos analisados. Além de uma breve análise de produtividade por autor, apresentando os principais e mais relevantes pesquisadores a respeito do assunto e respectivas publicações ao longo do tempo.

Para a pesquisa de trabalhos publicados foram utilizadas duas bases de dados acadêmicos, dentre as mais significativas em produção cientifica em meio acadêmico, a quais tem-se acesso, sendo elas: a Web of Science e a Scopus. Foi buscado produções anteriores ao mês de março de 2019, por um período de 20 anos. Como o objetivo é levantar o desenvolvimento de algoritmos para detecção de árvores até o presente momento, a inclusão do ano de 2019, ainda incompleto, não irá interferir no desenvolvimento do trabalho.

Para atingir o maior número possível de trabalhos relacionados, buscou-se a combinação do maior número de palavras chaves sobre o tema em questão, as palavras chaves utilizadas foram: "tree detection", "tree delineation", "tree count", "tree identification" "tree individual", "tree extration", "crown detection", "crown delineation", "crown identification" e combinações associadas com as palavras "LiDAR", "photogrammetry", "ALS" (Airborne Laser Scanning), "remote sensing" e "forest 
inventory". Assim, permitindo encontrar artigos publicados em inglês ou português, nas revistas indexadas nas bases utilizadas no presente estudo.

Dentre os principais assuntos discutidos, fez-se uma análise dos métodos empregados à identificação de árvores individuais, em busca de tendências na coleta e tratamento de dados, priorizando por técnicas genéricas para atendimento da demanda atual no manejo florestal.

Foram observadas as aplicações de algoritmos para detecção automática de árvores, técnicas de coleta de dados (aerofotogrametria ou LiDAR), espécies utilizadas, com destaque àquelas ocorrentes em reflorestamento no Brasil, idades e espaçamento. Mesmo que alguns estudos desconsideram algumas informações como o espaçamento, justamente por não tratarem de plantações florestais, indicando testes em florestas nativas, não foram descartados da análise com o objetivo de verificar os algoritmos empregados.

\section{RESULTADOS E DISCUSSÃO}

A busca genérica retornou 951 publicações cientificas em formato de artigo, destes inicialmente foram filtrados por área do conhecimento selecionado somente os relacionados a setor florestal, permanecendo assim 279 artigos. Desses, foram analisados todos os 279 artigos, mantendo somente os que se relacionavam com a identificação de árvores utilizando técnicas de sensoriamento remoto, definindo a amostra final em 188 documentos. O resumo dessas informações é apresentado na Tabela 1.

A Figura 1 apresenta a frequência das publicações no decorrer do tempo por ano, nos 20 anos. A primeira ocorrência foi em 2003 e no ano de 2019 foram contabilizadas apenas as publicações até março. De acordo com Ferreira (2010), a bibliometria apresenta três Leis consideradas básicas. A primeira Lei trata da produtividade dos periódicos (Lei de Bradford), que identifica qual periódico possui maiores tendências na publicação do tema de interesse.

A segunda Lei trata da produtividade dos autores (Lei de Lotka), interpretada como a probabilidade de produtividade, em que, o número de trabalhos publicados aumenta a probabilidade da publicação de novos trabalhos relacionados ao mesmo tema pelo autor. A terceira Lei se trata da frequência de ocorrência de palavras (Lei de Zipf), também, chamada da Lei do menor esforço, consiste na medição de frequência de palavras chave em trabalhos com assuntos correlacionados.
Tabela 1. Resumo da bibliometria, referente a publicações relevantes a contagem de árvores.

\begin{tabular}{cc}
\hline Descrição & Resultados \\
\hline Documentos publicados & 188 \\
\hline Fontes (Revistas) & 44 \\
Keywords Plus & 1278 \\
Palavras Chave - Autor & 521 \\
\hline Período & $2003-2019$ \\
\hline Média de citações por documento & 23,66 \\
Autores & 622 \\
\hline Autores citados & 856 \\
Autores únicos & 3 \\
\hline Multi-autoria & 619 \\
\hline Documentos de autores únicos & 5 \\
\hline Documentos por autor & 0,31 \\
\hline Autores por documento & 3,31 \\
\hline Coautores por documento & 4,55 \\
\hline Índice de colaboração & 3,38 \\
\hline Tipos de documento & \\
\hline Artigo & 152 \\
\hline Artigo em revista & 2 \\
\hline Artigo de conferências & 25 \\
\hline Errata & 1 \\
\hline Revisão & 8 \\
\hline
\end{tabular}

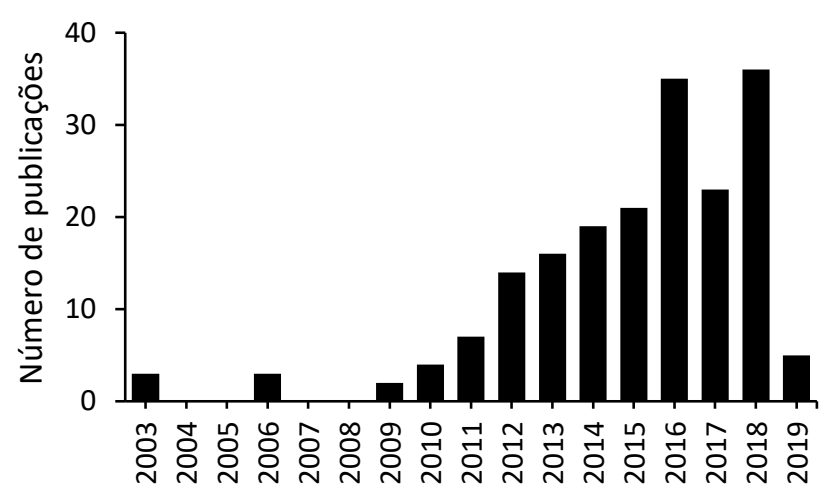

Figura 1. Frequência de publicação anual, referente a publicações sobre algoritmos de contagem de árvores.

De acordo com a Lei de Bradford, os três principais periódicos no quesito produtividade a tratar do tema em ordem decrescente são: Remote Sensing, Forests e ISPRS com 47, 14 e 13 publicações, respectivamente.

A interpretação da Lei de Lotka foi baseada no número de publicações por autor, dos quais, 78\% dos autores contam com somente uma publicação. Enquanto 14,3\% dos autores contam com duas publicações. O maior número de publicações por autor sobre o tema é de 9 trabalhos, em que, apenas $0,3 \%$ dos autores possuem este 
montante, que são Hyypp J. e Yu X. O brasileiro Silva C. A. possui 7 publicações, o único autor brasileiro com este número de publicações referido ao tema.

A Figura 2, apresenta o percentual do número de publicações por autor e a probabilidade de que autores aumentem o número de publicações relacionadas ao mesmo tema (linha pontilhada vermelha), esta probabilidade de publicações futuras por autor, representa a Lei de Lotka.

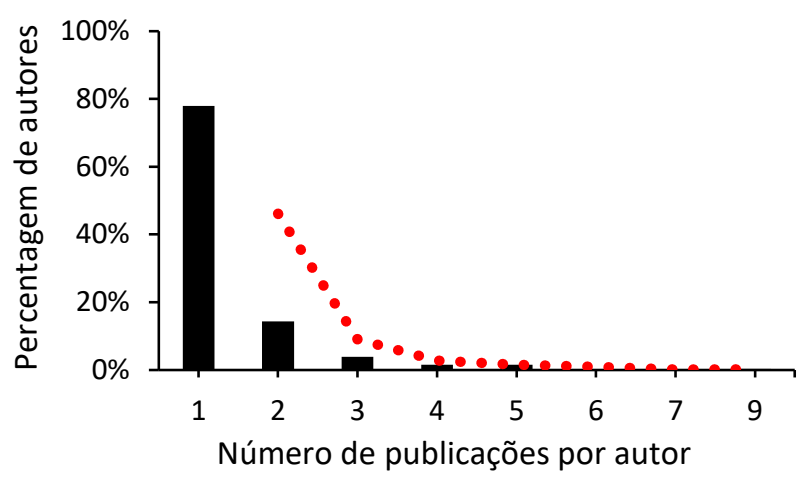

Figura 2. Representação da Lei de Lotka das publicações sobre algoritmos de contagem de árvores.

Baseado na Lei de Zipf, foram classificadas as principais palavras chaves de acordo com a frequência de ocorrência, quando maior a frequência maior a área que determinada palavra ocupa na nuvem de palavras, as palavras LiDAR, Forest Inventory, Remote Sensing, Airbone Laser Scanning e Segmentation são as mais frequentes nos trabalhos publicados. A Figura 3 apresenta uma nuvem de palavras chave seguindo a Lei de Zipf.

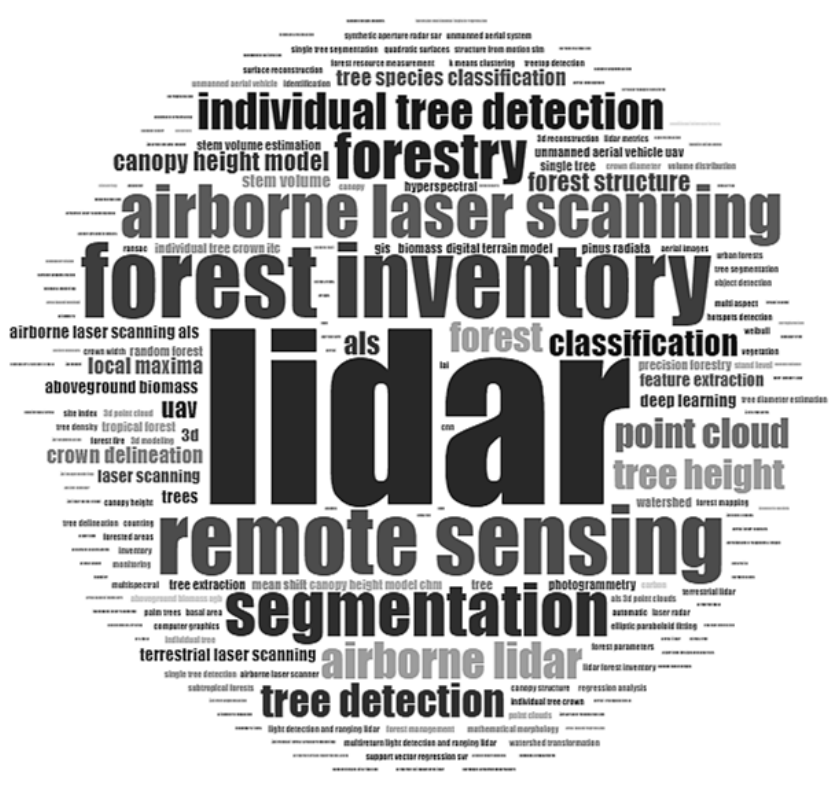

Figura 3. Nuvem de palavras-chave mais utilizadas, nos documentos analisados.
Os países que mais publicaram a respeito de identificação de árvores foram Estados Unidos da América com 128 publicações e China com 58 publicações. O Brasil é o quinto colocado no ranking com 31 publicações. A Figura 4 apresenta a intensidade de publicação por país e a relação estabelecida entre eles, por meio de citações entre trabalhos publicados.

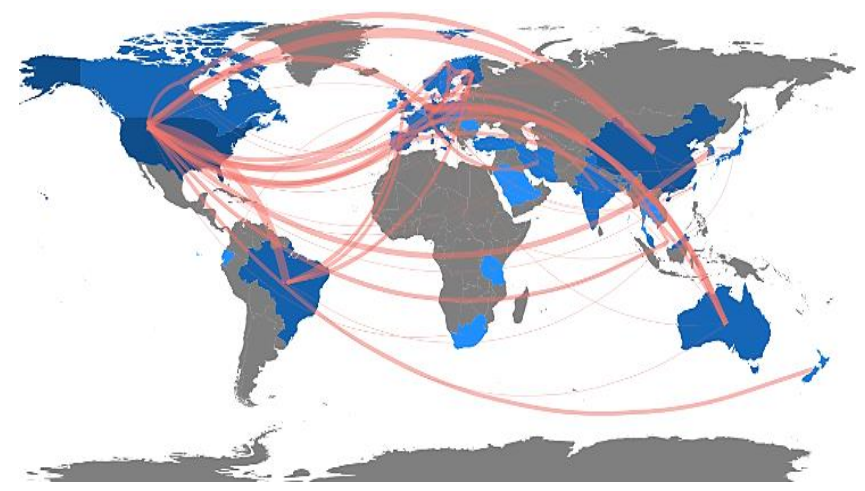

Figura 4. Distribuição espacial e relacionamentos das publicações sobre metodologias de detecção de árvores.

A Figura 5 mostra a produção ao longo do tempo dos principais autores, em que, o tamanho do ponto representa o número de publicações e a intensidade da cor o número de citações das publicações anuais. Observa-se que a produtividade e relevância das publicações no decorrer do tempo é dinâmica.

Com os documentos analisados, possibilitou-se a análise da produção tecnológica e científica relacionado a contagem de árvores. É observado diferentes técnicas de captura de dados, sensores, metodologias de contagem e validação, assim como, as particularidades de algumas espécies.

Atualmente, o uso de ARP oferecem uma nova perspectiva na coleta de dados espaciais ao setor florestal. Além de embarcarem sensores passivos, existem equipamentos capazes de acoplarem sistemas ativos como o LiDAR. Conforme resultados da revisão sistemática da literatura é possível observar que as ARP foram utilizadas em diversos estudos florestais voltados a individualização de árvores.

Hentz et al. (2018) utilizaram ARP na coleta de dados com sensor passivo com a finalidade de gerar nuvens de pontos tridimensionais, por correlação fotogramétrica em florestas de Eucalyptus spp. e Pinus taeda L. com idade de 1,5 anos, para posterior individualização baseada em segmentação de bacias hidrográficas e máximos locais atingindo um erro máximo de $6 \%$. 


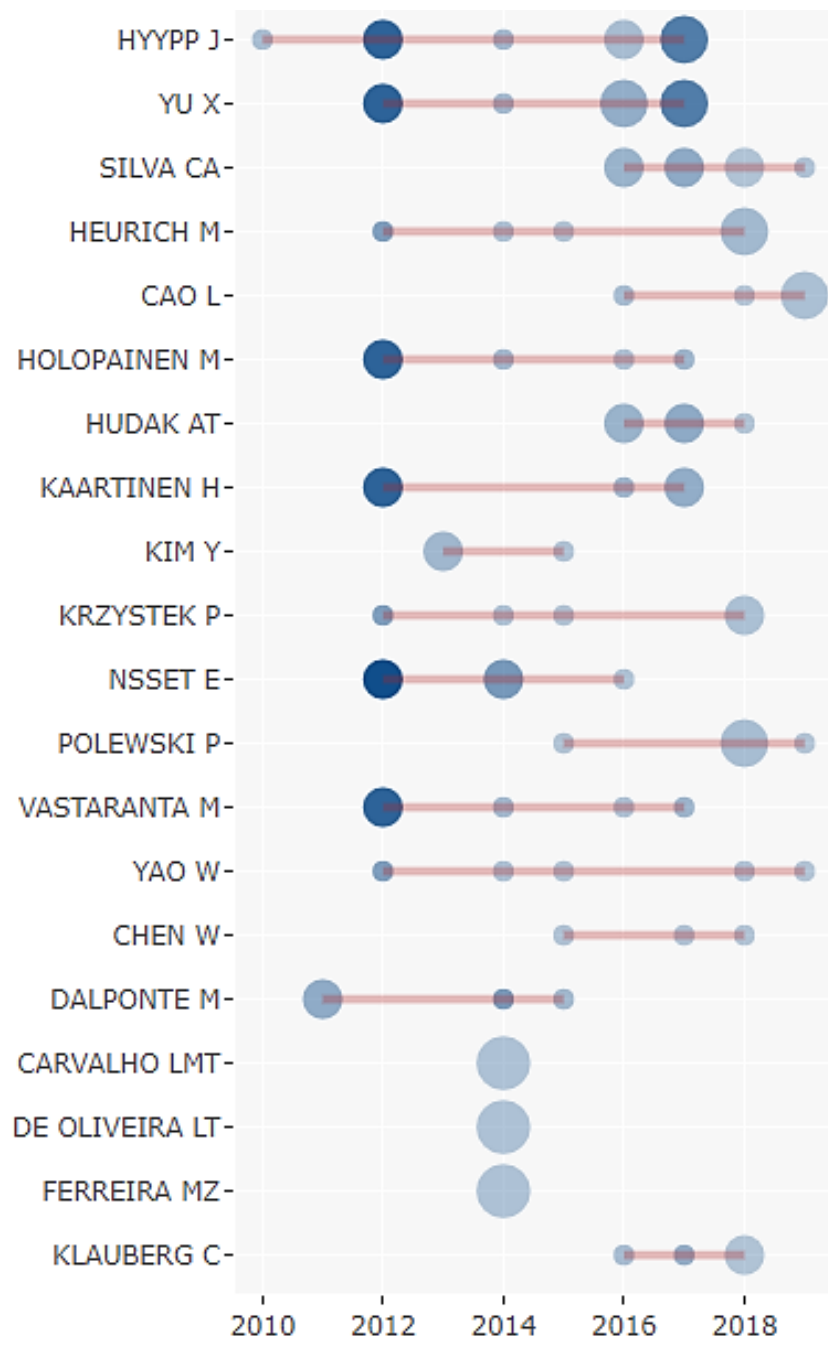

Figura 5. Produtividade por autor de publicações sobre metodologias de detecção de árvores, ao longo do tempo.

Jaakkola et al. (2017) também utilizaram um ARP na coleta de dados, porém, com um sensor ativo de baixo custo embarcado, atingindo uma taxa de detecção de $100 \%$ em árvores isoladas e dominantes, mostrando a viabilidade para o uso de ARP e sensores de baixo custo para as características especificas da floresta estudada, que consiste em uma floresta boreal manejada de idade não determinada onde as principais espécies dominantes são Pinus sylvestris e Picea abies.

Koc-san et al. (2018) implementaram a extração automática de árvores cítricas (Citrus sinensis L. Osbeck), fazendo uso de modelos digital de superfície obtidos por imagens tomadas com ARP, aplicando limiarização sequencial, detecção de borda de Canny e a transformada circular de Hough, atingindo uma acurácia superior a $80 \%$.

Panagiotidis et al. (2017) utilizaram imagens tomadas por ARP para reconstrução da superfície de dossel florestal aplicando a técnica de reconstrução Structure for motion
(SfM), em talhões de diferentes idades de Picea abies L., Larix decidua Mill., Pinus sylvestris L. e Betula pendula Roth, para a obtenção da altura aplicou o algoritmo de máximas locais e para delimitação das copas a segmentação inversa de bacias hidrográficas.

Thiel et al. (2017) procederam a detecção de árvores de espécies coníferas e idade variável, com base em nuvem de pontos extraída de imagens tomadas por ARP, aplicando ao modelo digital de copas o algoritmo de máximas locais com janela variável em função da altura, atingindo 93\% de acerto na identificação de árvores com o uso da nuvem de pontos ARP e 78\% utilizando dados LiDAR.

Wallace et al. (2016), com a utilização de sensores passivos e ativos embarcados em uma ARP, compararam o emprego da técnica SfM e de dados ALS para a contagem de árvores em uma floresta de Eucalyptus ssp. com idade variável.

Wallace et al. (2016) verificaram que a nuvem de pontos obtida por SfM propicia qualidade inferior na modelagem do terreno, consequentemente na determinação da altura e na estimativa de cobertura do dossel, mesmo o ALS oferecendo estimativas mais precisas, nuvem de pontos obtidas via SfM podem ser consideradas com uma alternativa para estimativas florestais de baixo custo.

Yan et al. (2018), com uso de um ARP embarcado com um ALS de baixo custo, realizaram a segmentação de árvores individuais em nuvem de pontos normalizada, classificada e de alta densidade, a extração deu-se por segmentação de corte normalizado, de modo interativo em função de curvas de forma em perfil, atingindo exatidão de $90 \%$.

Em povoamentos de Eucalyptus ssp. as metodologias identificação de árvores por sensoriamento remoto apresentaram-se satisfatória em diversos estudos, com idades e densidade diferentes, como foi examinado.

Tesfamichael et al. (2009) pesquisaram alternativas para a individualização de árvores em plantios de Eucalyptus ssp. com uso de dados LiDAR aerotransportado com retorno discreto, gerando modelos de copa com diferentes resoluções espaciais $(0,2 \mathrm{~m}, 0,5 \mathrm{~m}$ e $1 \mathrm{~m})$, com diferentes densidades de ponto ALS $\left(1 \mathrm{p} / \mathrm{m}^{2}, 3 \mathrm{p} / \mathrm{m}^{2}\right.$ e 5 $\mathrm{p} / \mathrm{m}^{2}$ ) e janela fixa em função das características médias da floresta estudada.

No estudo de Tesfamichael et al. (2009), para estimativa do número de indivíduos, todas as alternativas de processamento subestimaram o número real de indivíduos, a melhor resolução espacial do modelo digital de copas foi definida em $0,2 \mathrm{~m}$. A densidade de pontos com diferenças mínimas quando comparadas as duas maiores 
densidades, os autores consideraram que nuvens mais densas que $3 \mathrm{p} / \mathrm{m}^{2}$ não trazem muitos benefícios, visto que as estimativas atingidas não ultrapassaram $82 \%$ de acurácia.

Cosenza et al. (2018) realizaram a contagem e estimativa de volume para povoamentos florestais de Eucalyptus ssp. de 4 a 7 anos de idade, aplicando o algoritmo de máximos locais atingindo níveis satisfatórios na contagem de árvores, desde que os resultados sejam calibrados com inventários em campo.

Oliveira et al. (2014) analisaram a influência da idade em talhões de Eucalyptus ssp., na contagem automática de árvores, foram testadas as idades 3, 5 e 7 anos, utilizado o primeiro retorno dos dados ALS e verificação em imagens de alta resolução. Em que, não foram observadas diferenças significativas entre as idades, somente uma recomendação aos talhões de 7 anos de idade, no qual consiste em utilizar apenas os dados acima de 10 metros de altura, atingindo assim resultados satisfatórios referente a contagem de árvores em todos os talhões testados.

De Oliveira et al. (2012) executaram a contagem de árvores em povoamento de Eucalyptus ssp. com algoritmo de máximas locais com janelas fixas de busca, com base em dados ALS, modelaram-se as copas por triangulação irregular e interpolação com ponderação inversa à distância, atingiram uma acurácia de 97,36\% verificados em uma imagem orbital Quickbird.

Testes em povoamentos de Eucalyptus ssp. se apresentaram satisfatórios na totalidade de estudos apresentados, independentemente da idade, espaçamento ou densidade de pontos, assim como, o emprego quase majoritário do algoritmo de máximos locais.

A frequência de estudos em povoamentos de Pinus ssp. se apresentaram menos constante, quando comparada a estudos relacionados ao Eucalyptus ssp., assim como os algoritmos utilizados não seguem uma metodologia padrão, são testadas diversas alternativas, conforme apresentados nos estudos a seguir.

Straub \& Koch (2011) identificaram manualmente a coroa e o ápice das copas de parcelas instaladas em povoamento de Pinus sylvestris, utilizando dados LiDAR aerotransportado e sensores multiespectrais, para estimativas de volume de tronco com base na altura das árvores e diâmetro de copas.

Kathuria et al. (2016) desenvolveram um algoritmo de identificação de árvores baseado em máximos locais com raio de busca dinâmico em função da vizinhança dos pontos LiDAR aerotransportado, para a espécie Pinus radiata estimando o número de árvores com um erro médio quadrático de $5,7 \%$, em dados de baixa densidade ( 2 pontos $/ \mathrm{m}^{2}$ ), porém, computacionalmente exigente.

Silva et al. (2016b) fizeram uso de classificador RF k-NN (Random Forest e k-Nearest Neighbor) para detecção individual de árvores em povoamento de Pinus palustres Mill, baseado no modelo de copas proveniente de dados LiDAR aerotransportado, atingindo alta precisão chegando a $96 \%$ de acurácia em florestas com cobertura de dossel menor que $70 \%$.

Chen \& Zhu (2013) integraram uma plataforma de sistema de informação geográfica ao tratamento de dados LiDAR, na qual, faz uso de segmentação de bacias hidrográficas controlada por marcador, com testes em povoamentos de Pinus radiata, denso e não desbastado, atingindo precisão de $1,87 \mathrm{~m}$ na posição das árvores identificadas.

Para povoamentos de Pinus ssp. não existe um domínio de apenas um algoritmo de detecção automática de árvores, pois, são utilizados diferentes algoritmos, assim como, diferentes pré-processamentos no tratamento das nuvens de pontos LiDAR. Muitos estudos para Pinus ssp. não atingiram níveis satisfatórios de detecção de árvores como foi observado para Eucalyptus ssp. por apresentarem influências da idade, espaçamento, densidade de pontos LiDAR.

Tanhuanpaa et al. (2016), com base em dados de sensores orbitais e com resolução espacial de 50 centímetros, aplicaram a segmentação de bacias hidrográficas a o modelo de copas em florestas boreais, atingindo detecções de $26 \%$ até $140 \%$, mesmo a resolução espacial sendo considerada suficiente, o efeito do sombreamento das árvores dominantes prejudicou a execução da detecção.

Nevalainen et al. (2017), com imagens multiespectrais coletadas por ARP em floretas mistas com espécies e idades diferentes e espaçamento não regulares, aplicaram classificadores de imagens em que se atingiu uma acurácia de $95 \%$, e a identificação de árvores com base na nuvem de pontos fotogramétrica variou de $40 \%$ a $95 \%$ em dependência das diferentes características dos povoamentos estudados.

Mohan et al. (2017) implementaram a identificação automática de árvores em floresta de coníferas mistas de dossel aberto, com base em modelo de copas derivado de nuvem de pontos fotogramétricos obtidos por ARP, utilizando o algoritmo de máximos locais, atingindo a acurácia de $85 \%$.

Lin et al. (2018) apresentaram que as nuvens de pontos obtidas por imagens aéreas obliquas são limitadas pela 
relativamente fraca capacidade de penetração da luz natural, bem como, apresentaram que as fotografias capturadas pela câmera digital em um ARP são mais adequadas para obter os dados de nuvens de pontos em uma floresta subalpina relativamente esparsa.

A detecção de árvores por meio de dados provenientes de sensores passivos mostrou-se satisfatória desde que observado o objetivo e o tipo de povoamento a ser empregado, por exemplo, árvores suprimidas não serão identificadas quando utilizados estes dados. Assim como, estudos mostraram uma incerteza maior na determinação de altura das árvores, quando comparada a identificações utilizando dados LiDAR.

Alternativas de otimização aos dados LiDAR ou estudo de novas possibilidades em função do avanço da tecnologia está sendo desenvolvidos com a combinação da dados LiDAR e RGB, dados LiDAR multiespectrais e otimização de algoritmos. Kaartinen et al. (2012) demonstraram que ainda há trabalho significativo a ser realizado quando se trata de detecção de árvores por meio de dados LiDAR.

Deng et al. (2016) delinearam individualmente árvores em povoamentos de Pinus densiflora, Chamaecyparis obtusa e Larix kaempferi, em que, se verificaram uma otimização de até $14,1 \%$ na classificação de indivíduos quando combinado dados LiDAR com imagens RGB.

Yu et al. (2017) investigaram o potencial de um LiDAR aerotransportado multiespectral em povoamentos de Pinus sylvestris, Picea abies e Betula sp. atingindo uma acurácia de $91,9 \%$ para árvores consideradas isoladas ou dominantes no povoamento em estudo.

As informações obtidas por meio de dados LiDAR possibilitam a análise de múltiplos retornos, o que viabilizam a identificação de árvores suprimidas pela competição na floresta. São menos exigentes computacionalmente no quesito geração de nuvem de pontos, são mais precisos posicionalmente e proporcionam uma acurácia superior na altura das árvores em relação a técnicas que utilizam imagens.

Gorgens et al. (2015) compararam dois modos de definição da janela de busca para o algoritmo de máximos locais, sendo um modo proporcional à altura do modelo digital de alturas e outro inversamente proporcional à altura, em povoamentos de Picea engelmannii Parry e Abies lasiocarpa Nutt, com levantamento ALS de retornos discretos com densidade de 2 pontos $/ \mathrm{m}^{2}$ e small-footprint. O filtro subestimou o número de árvores em até $92 \%$ do total, porém, a relação inversa à altura apresentou melhores resultados principalmente em regiões com alta densidade de árvores.

Kaartinen et al. (2012) realizaram um estudo adicional ao benchmarking "extração de árvores" (2005 - 2008) somando mais 4 algoritmos ao conjunto de 9 algoritmos testados no estudo anterior e um procedimento de contagem manual, testes estes realizados em povoamentos de Pinus sylvestris, Picea abies e Betula sp. levantamento ALS com densidade de pontos de 2, 4 e 8 pontos por $\mathrm{m}^{2}$. Dentre todos os testes aplicados, o algoritmo de máximo local foi uma das melhores técnicas aplicadas ao estudo, atingindo $102 \%$ para a estimativa do número de indivíduos dominantes.

Conforme Kaartinen et al. (2012), a densidade de pontos ótima é dependente do tamanho da árvore, densidade de povoamento da floresta, espera-se que o aumento da densidade de pontos LiDAR aumente a precisão da detecção de árvores, consequentemente, o custo de aquisição de dados e armazenamento.

Wang et al. (2016) constataram que diferente dos estudos anteriores a densidade da nuvem de pontos é fator altamente influente na individualização de árvores em povoamentos florestais.

Ainda existem divergências na questão da densidade da nuvem de pontos ideais, entretanto, estudos recentes apontaram a ocorrência de uma maior densidade de pontos como fator positivo na detecção de árvores em dados LiDAR.

\section{CONCLUSÕES}

Com base nas publicações analisadas é possível estabelecer o estado do desenvolvimento tecnológico e científico para a individualização de árvores em povoamentos florestais. Nos dois últimos anos, o uso de ARP no levantamento de informações florestais, é mostrado usual com sensores passivos e ativos para estudos de detecção de árvores individuais apresentando acurácias acima de $80 \%$, grandeza considerada como satisfatória, principalmente para povoamentos de Eucalyptus ssp. do que para povoamentos de Pinus spp.

No decorrer da pesquisa não é identificado uma metodologia padrão em função de espécies, espaçamentos, tipo de aquisição de dados, préprocessamentos e a implementação de algoritmos. Mas tendências voltadas a estudos baseados em modelos digitais de altura, segmentação de bacias hidrográficas tem considerável satisfação quando empregado o algoritmo de máximos locais para as diversas espécies e tipos de obtenção de dados citados.

A detecção de árvores automatizada ainda se apresenta como uma tarefa desafiadora e importante na estimativa florestal a nível de indivíduos de um 
povoamento, proporciona mais acurácia as estimativas e indícios de diminuição de custos relacionados a trabalhos de campo, porém, dependente ainda, de uma análise orçamentária detalhada das atividades para confirmação da informação.

\section{REFERÊNCIAS}

BIOLCHINI, J.C.A. et al. Scientific research ontology to support systematic review in software engineering. Advanced Engineering Informatics, v.21, n.2, p.133-151, 2007.

CHEN, Y.; ZHU, X. An integrated GIS tool for automatic forest inventory estimates of Pinus radiata from LiDAR data. GIScience and Remote Sensing, v.50, n.6, p.667-689, 2013.

COSENZA, D.N. et al. Airborne laser scanning applied to eucalyptus stand inventory at individual tree level. Pesquisa Agropecuária Brasileira, v.53, p.1373-1382, 2018.

DE OLIVEIRA, L.T. et al. Application of lidar to forest inventory for tree count in stands of Eucalyptus sp. Cerne, v.18, n.18, p.175184, 2012.

DENG, S.Q. et al. Comparison of tree species classifications at the individual tree level by combining ALS data and RGB images using different algorithms. Remote Sensing, v.8, n.22, p.1-22, 2016.

FERREIRA, A.G.C. Bibliometrics in the evaluation of scientific journals. DataGramaZero, v.11, n.3, p.1-13, 2010.

GORGENS, E.B. et al. Individual tree identification in airborne laser data by inverse search window. Cerne, v.21, n.1, p.91-96, 2015.

HENTZ, Â.M.K. et al. Estimating forest uniformity in Eucalyptus spp. and Pinus taeda L. stands using field measurements and structure from motion point clouds generated from unmanned aerial vehicle (UAV) data collection. Forest Systems, v.27, p.1-17, 2018.

JAAKKOLA, A. et al. Autonomous collection of forest field reference-the outlook and a first step with UAV laser scanning. Remote Sensing, v.9, n.12, p.1-12, 2017.

KAARTINEN, H. et al. An international comparison of individual tree detection and extraction using airborne laser scanning. Remote Sensing, v.4, n.4, p.950-974, 2012.

KATHURIA, A. et al. Development of an automated individual tree detection model using point cloud LiDAR data for accurate tree counts in a Pinus radiata plantation. Australian Forestry, v.2, n.79, p.126-136, 2016.

KOC-SAN, D. et al. Automatic citrus tree extraction from UAV images and digital surface models using circular Hough transform. Computers and Electronics in Agriculture, v.150, p.289-301, 2018.

LIN, J.Y. et al. Aboveground tree biomass estimation of sparse subalpine coniferous forest with UAV oblique photography. Remote Sensing, v.10, n.19, p.1-19, 2018.
MOHAN, M. et al. Individual tree detection from unmanned aerial vehicle (UAV) derived canopy height model in an open canopy mixed conifer forest. Forests, v.8, n.17, p.1-17, 2017.

NEVALAINEN, O. et al. Individual tree detection and classification with UAV-based photogrammetric point clouds and hyperspectral imaging. Remote Sensing, v.9, n.34, p.1-34, 2017.

OK, A.O.; OZDARICI-OK, A. 2-D delineation of individual citrus trees from UAV-based dense photogrammetric surface models. International Journal of Digital Earth, v.11, p.583-608, 2018.

OLIVEIRA, L. et al. Influência da idade na contagem de árvores de Eucalyptus sp. com dados de lidar. Cerne, v.20, n.4, p.557-565, 2014.

PACKALÉN, P. et al. ALS-based estimation of plot volume and site index in a eucalyptus plantation with a nonlinear mixed-effect model that accounts for the clone effect. Annals of Forest Science, v.6, n.68, p.1085-1092, 2011.

PANAGIOTIDIS, D. et al. Determining tree height and crown diameter from high-resolution UAV imagery. International Journal of Remote Sensing, n.38, p.2392-2410, 2017.

SILVA, C.A. et al. A principal component approach for predicting the stem volume in Eucalyptus plantations in Brazil using airborne LiDAR data. Forestry, v.89, n.4, p.422-433, 2016a.

SILVA, C.A. et al. Imputation of Individual Longleaf Pine (Pinus palustris Mill.) Tree Attributes from Field and LiDAR Data. Canadian Journal of Remote Sensing, v.42, n.5, p.554-573, 2016b.

STRAUB, C.; KOCH, B. Estimating single tree stem volume of Pinus sylvestris using airborne laser scanner and multispectral line scanner data. Remote Sensing, v.5, n.3, p.929-944, 2011.

TANHUANPAA, T. et al. Evaluating the performance of highaltitude aerial image-based digital surface models in detecting individual tree crowns in mature boreal forests. Forests, v.7, n.17, p.1-17, 2016.

TESFAMICHAEL, S.G. et al. A semi-variogram approach for estimating stems per hectare in Eucalyptus grandis plantations using discrete-return lidar height data. Forest Ecology and Management, v.258, n.7, p.1188-1199, 2009.

THIEL, C.; SCHMULLIUS, C. Comparison of UAV photograph-based and airborne lidar-based point clouds over forest from a forestry application perspective. International Journal of Remote Sensing, v.38, n.8, p.2411-2426, 2017.

WALLACE, L. et al. Assessment of forest structure using two UAV techniques: a comparison of airborne laser scanning and structure from motion (SFM) point clouds. Forests, v.7, n.6, p.116, 2016.

WANG, Y.S. et al. International benchmarking of the individual tree detection methods for modeling 3-d canopy structure for silviculture and forest ecology using airborne laser scanning. IEEE Transactions on Geoscience and Remote Sensing, v.54, n.9, p.5011-5027, 2016. 
WEBSTER, J.; WATSON, J.T. Analyzing the past to prepare for the future: writing a literature review. MIS Quarterly \& The Society for Information Management, v.26, n.2, pp.13-23, 2002.

YAN, W. et al. An automated hierarchical approach for threedimensional segmentation of single trees using UAV LiDAR data. Remote Sensing, v.10, p.1-16, 2018.

YU, X.W. et al. Single-sensor solution to tree species classification using multispectral airborne laser scanning. Remote Sensing, v.9, n.16, p.1-16, 2017.

Recebido em 16-05-2019

Aceito em 25-08-2019 